Series A

I. MATHEMATICA

527

\title{
ÜBER DEN SATZ VON STOKES
}

rox

PETER HERMANN

HELSINKI 1973

S U O M A L I NEN TIEDEAKATEMIA

https://doi.org/10.5186/aasfm.1973.527 
Copyright (C) 1972 by Academia Scientiarum Fennica ISBN 951-41-0068-9

Am 8. September 1972 vorgelegt ron Rolf Nevarlisia 


\section{Einleitung}

F. und R. Nevanlinna führen in [4], III.2.6, eine Verallgemeinerung der alternierenden (Cartanschen) Ableitung von Differentialformen (dort Rotor genannt) ein und beweisen damit den Satz von Stokes für Simplexe ([4], III.2.7). Daran anschließend ([4], III.2.8) beginnen sie einen zweiten Beweis des Stokesschen Satzes, dessen Ansatz im Grunde genommen näherliegt als die Idee ihres zuerst zitierten Beweises. Dieser zweite Beweis wird jedoch nicht zu Ende geführt, weil das dabei benötigte gleichmäßige Verschwinden eines gewissen Restgliedes "im allgemeinen nicht direkt zu ersehen ist

Ziel dieser Note ist die Fortführung jenes Beweises durch eingehendere Untersuchung des fraglichen Restgliedes.

Genau diese Aufgabe hat sich bereits T. Klemola in [2] gestellt. Allerdings ändert er die Nevanlinnasche Definition des Rotors $a b^{1}$ ), wodurch der Beweis des Satzes von Stokes erheblich erleichtert wird. Zwar impliziert die Voraussetzung bei Nevanlinna (Stetigkeit des Nevanlinnaschen Rotors) die Existenz und Stetigkeit des Rotors im Sinne von Klemola, doch ergibt sich dies erst als Folgerung des zu beweisenden Stokesschen Satzes.

Wir führen die nachstehende Untersuchung mit einer in [1] behandelten, dem Nevanlinnaschen Rotor entsprechenden Verallgemeinerung der alternierenden Ableitung durch. Auf diese Weise vermeiden wir zusätzliche Überlegungen im Zusammenhang mit den bei Nevanlinna erforderlichen Zerlegungen eines Simplex in Teilsimplexe mit gleichmäßig beschränktem Regularitätsindex, da jetzt Würfel an die Stelle der Simplexe treten. Der hier dargestellte Beweis läßt sich jedoch ohne weiteres, etwa mit Hilfe der ron H. Whitney ([5], Appendix II.4) angegebenen "Normalunterteilung" ("standard subdivision") eines Simplex, auf die Nevanlinnasche Theorie übertragen.

Zur Vereinfachung der formalen Darstellung beschränken wir uns auf $(n-1)$-Differentialformen im $n$-dimensionalen reellen Zahlenraum $\boldsymbol{R}^{n}$; zur Herleitung des Stokesschen Satzes für Differentialformen in einem

1) Man vergleiche ${ }^{2}$ ). Die Definition von Klemola ist nicht mit der Nevanlinnaschen äquivalent, vielmehr folgt aus der Existenz des Rotors in seinem Sinn, daß der Nevanlinnasche Rotor existiert und stetig ist. 
beliebigen reellen linearen Raum mit Skalarprodukt kann die hier verfolgte Idee aber auch ausgenutzt werden.

In dem Fall, daß die vorgelegte Differentialform Koeffizientenfunktionen mit Werten in einem reellen oder komplexen linearen Raum endlicher Dimension besitzt, folgt das gleichmäßige Verschwinden des in Rede stehenden Restgliedes (oder gleich der Stokessche Satz) auch aus einer von Cl. Müller [3] gefundenen Verallgemeinerung des Mittelwertsatzes der Differentialrechnung (man vgl. [1], 5.1, oder [1a], 4.1).

\section{Definitionen und Bezeichnungen}

Wenn wir im folgenden von $n$-dimensionalen Würfeln sprechen, verstehen wir darunter stets kompakte $n$-dimensionale (nicht notwendigerweise achsenparallele) Würfel des $\boldsymbol{R}^{n}(n \geqq 1)$. Den euklidischen Inhalt eines solchen Würfels $W \subset \boldsymbol{R}^{n}$ bezeichnen wir mit $I(W)$. Ton den rorkommenden $(n-1)$-Differentialformen nehmen wir an, daß ihre Koeffizientenfunktionen Werte in einem reellen oder komplexen Banachraum $(B,\|\cdot\|)$ besitzen.

Definition 1.1. Es sei $\omega$ eine in einer Umgebung $U\left(x^{*}\right)$ ion $x^{*} \in \mathbf{R}^{n}$ stetige $(n-1)$-Differentialform. Falls dann ein solches $f\left(x^{*}\right) \in B$ existiert. daß für alle n-dimensionalen Würfel $W$ mit $W \subset U\left(x^{*}\right)$ eine Zerlegung

$$
\int_{\partial W} \omega=\int_{W} f\left(x^{*}\right) d x_{1} \wedge \ldots \wedge d x_{n}+I(W) r\left(x^{*}, W\right)
$$

besteht, wobei es zu jedtem $\varepsilon>0$ ein $\delta\left(\varepsilon, x^{*}\right)>0$ so gibt, da $r\left(x^{*}, W\right)$ $<\varepsilon$ für alle $W \subset\left\{x \in \boldsymbol{R}^{n}|| x-x^{*} \mid<\delta\left(\varepsilon, x^{*}\right)\right\}$ mit $\left.x^{*} \in W^{2}\right)$ gilt, so heißt $\omega$ in $x^{*}$ elementar derivierbar.

Ist $\omega$ in $x^{*}$ elementar derivierbar, so ist $f\left(x^{*}\right)$ eindeutig bestimmt.

Hinreichend, aber nicht notwendig zur elementaren Derivierbarkeit der stetigen Differentialform $\omega$ ist die Differenzierbarkeit ron $\omega$ in $x^{*}$.

Wenn $\omega$ in allen Punkten einer Menge $M \subseteq \mathbf{R}^{n}$ elementar derivierbar ist, setzen wir $f: M \ni x \mapsto f(x) \in B$ und definieren

$$
d \omega:=f d x_{1} \wedge \ldots \wedge d x_{n} .
$$

Die $n$-Differentialform $d \omega$ stimmt mit der üblicherweise erklärten Cartanschen Ableitung von $\omega$ überein, falls $\omega$ differenzierbar ist.

2) Die der Eigenschaft $x^{*} \in W$ in der Nevanlinnaschen Theorie entsprechende, Bedingung läßt Klemola bei seiner Definition ([2], 10) weg. 


\section{Der Satz von Stokes}

Zunächst wollen wir die gleich benötigten Eigenschaften des Restgliedes $r\left(x^{*}, W\right)$ aus Dafinition 1.1 beweisen. Dazu bemerken wir, daß wegen

$$
I(W)=\int_{W} d x_{1} \wedge \ldots \wedge d x_{n}
$$

die Identität

$$
r\left(x^{*}, W\right)=\frac{1}{I(W)} \int_{\partial W} \omega-f\left(x^{*}\right)
$$

besteht.

Lemma 2.1. Die (n-1)-Differentialform $\omega$ sei in allen Punkten des n-dimensionalen Würfels $W_{0}$ elementar derivierbar. Wird $W_{0}$ in $N$ inhaltsgleiche Teilwürfel $W_{i}(i=1, \ldots, N)$ zerlegt und dann je ein Punkt. $x_{i} \in W_{i}(i=0,1, \ldots, N)$ gewählt, so gilt

$$
r\left(x_{0}, W_{0}\right)=\frac{1}{N} \sum_{i=1}^{N} r\left(x_{i}, W_{i}\right)+\frac{1}{N} \sum_{i=1}^{N}\left(f\left(x_{i}\right)-f\left(x_{0}\right)\right) .
$$

Beweis. Ersetzen wir in (2.1) jeweils $x^{*}$ durch $x_{i}$ und $W$ durch $W_{i} \quad(i=0,1, \ldots, N)$, so läßt sich die Behauptung durch einfaches Nachrechnen verifizieren, wenn wir noch die Additivität des Randintegrals und des Inhalts

$$
\int_{\partial W_{0}} \omega=\sum_{i=1}^{N} \int_{\partial W_{i}} \omega \quad \text { bzw. } I\left(W_{0}\right)=N I\left(W_{i}\right) \quad(i=1, \ldots, N)
$$

berücksichtigen.

Lemma 2.2. Die Voraussetzungen von Lemma 2.1 seiєn erfüllt. Gilt dann

$$
\left\|r\left(x_{0}, W_{0}\right)\right\| \geqq C,
$$

so besteht für mindestens ein $i_{1} \in\{1, \ldots, N\}$ die Abschätzung

$$
\left\|r\left(x_{i_{1}}, W_{i_{1}}\right)\right\| \geqq C-\max _{j=1, \ldots, N} \| f\left(x_{j}\right)-f\left(x_{0}\right) .
$$

Beweis. Wäre die Behauptung falsch, dann hätten wir für alle $i=1$, $\ldots, N$

$$
\left\|r\left(x_{i}, W_{i}\right)\right\|<C-\max _{j=1, \ldots, N} \| f\left(x_{j}\right)--f\left(x_{0}\right)
$$

und damit nach Lemma 2.1 


$$
\begin{aligned}
& \left\|r\left(x_{0}, W_{0}\right)\right\| \leqq \max _{i=1, \ldots, N}\left\|r\left(x_{i}, W_{i}\right)\right\|+\max _{i=1, \ldots, N}\left\|f\left(x_{i}^{\prime}\right)-f\left(x_{0}\right)\right\| \\
& <C-\max _{j=1, \ldots, N}\left\|f\left(x_{j}\right)-f\left(x_{0}\right)\right\|+\max _{i=1, \ldots, N} \| f\left(x_{i}\right)-f\left(x_{0}\right)=C
\end{aligned}
$$

im Widerspruch zur Voraussetzung.

Mit Hilfe von Lemma 2.2 beweisen wir den Satz von Stokes:

Satz 2.3. Die (n-1)-Differentialform (') sei in allen Punkten des ndimensionalen Würfels $W$ elementar derivierbar; darüberhinaus sei d(') in $W$ stetig. Dann gilt

$$
\int_{\partial W} \omega=\int_{W} d(\omega)
$$

Bewejs. Wir zerlegen $W$ durch Halbieren der Kanten in $2^{n}$ inhaltsgleiche Teilwürfel $W_{1 i}\left(i=1, \ldots, 2^{n}\right)$. Dann halbieren wir die Kanten jedes Teilwürfels, wodurch eine Zerlegung von $W$ in $\left(2^{n}\right)^{2}$ inhaltsgleiche Teilwürfel $W_{2 i}\left(i=1, \ldots,\left(2^{n}\right)^{2}\right)$ entsteht. Tterieren wir diesen Torgang, so gewinnen wir eine Zerlegung von $\Pi^{r}$ in $2^{n r}(v=1,2, \ldots)$ Teilwürfel $W_{r i}\left(i=1, \ldots, 2^{n v}\right)$, wobei

$$
I\left(W_{v i}\right)=\frac{1}{2^{n}} I\left(W^{-}\right)
$$

für $y \rightarrow \infty$ gegen Null strebt.

Wir wählen je ein $x_{v i} \in W_{v i}$ beliebig. Insbesondere in den Punkten $x_{v i}$ ist $\omega$ elementar derivierbar, so daß wir nach Definition 1.1

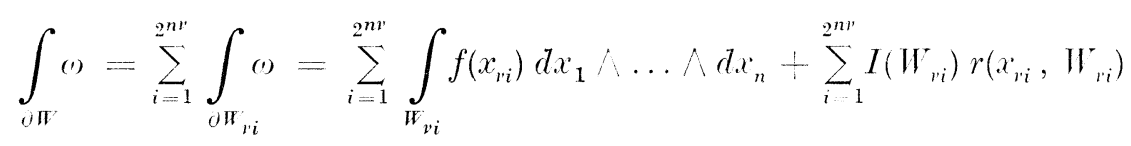

für jedes $v \in N:=\{1,2, \ldots\}$ erhalten. Auf Grund der Stetigkeit von $d \omega=f d x_{1} \wedge \ldots \wedge d x_{n}$ in $W$ konvergiert die vorletzte Summe für $v \rightarrow \infty$ gegen das Integral ron $d(1)$ über $W$. Die letzte Summe schätzen wir durch

$$
\| \sum_{i=1}^{2^{n v}} I\left(W_{v i}\right) r\left(x_{v i}, W_{v i}\right) \leqq \max _{i=1 \ldots .2^{n v}} r\left(r_{v i}, W_{v i}\right) \sum_{i=1}^{2^{n i}} I\left(W_{v i}\right)
$$

ab, wobei

$$
\sum_{i=1}^{2^{n v}} \mid I\left(W_{v i}\right)^{\prime}=I\left(\mathrm{H}^{*}\right)
$$

gilt. Daher bleibt zu zeigen, daß es zu jedem $\varepsilon>0$ ein solches $N^{\top}(\varepsilon)$ gibt, daß 


$$
\max _{i=1, \ldots, 2^{n v}} \| r\left(x_{v i}, W_{v i}\right)<\varepsilon
$$

für alle $v>N^{\top}(\varepsilon)$ ist.

Die Annahme, daß diese Behauptung falsch sei, soll zum Widerspruch geführt werden. Es gebe also ein $\varepsilon_{0}>0$ und eine Folge natürlicher Zahlen $v^{\prime} \rightarrow \infty$ mit

$$
\max _{i=1, \ldots, 2^{n v^{\prime}}} \| r\left(x_{v^{\prime} i}, W_{v^{\prime} i}^{r^{\prime}}\right)_{i} \geqq \varepsilon_{0}
$$

für alle $v^{\prime}$. Wegen der gleichmäßigen Stetigkeit von

$$
d \omega=f d x_{1} \wedge \ldots \wedge d x_{n}
$$

in $W$ läßt sich ein $v_{0}^{\prime}$ aus der Menge der Zahlen $v^{\prime}$ so wählen, daß

$$
\sup _{x, y \in W_{v_{0}^{\prime} i}} \| f(x)-f(y) \leqq \frac{1}{3} \varepsilon_{0}
$$

für alle $i=1, \ldots, 2^{n n_{0}^{\prime}}$ gilt. Zu $r_{0}^{\prime}$ gibt es ein $i_{0} \in\left\{1, \ldots, 2^{n r_{0}^{\prime}}\right\}$ mit

$$
\| r\left(x_{r_{0}, i_{0}}, W_{v_{0}^{\prime} i_{0}}\right)=\max _{i=1, \ldots, 2^{2} r_{0}^{\prime}} r\left(x_{r^{\prime} i}, W_{r_{n}^{\prime} i}\right) \geqq \varepsilon_{0} .
$$

Wir bestimmen zu jedem $v \in N$ ein $k_{v} \in N$ mit $k_{v}>k_{v-1}, \quad k_{1}>v_{0}^{\prime}$ derart, daß für alle $i=1, \ldots, 2^{n k_{r}}$.

$$
\sup _{x, y \in W_{k_{v} i}} \| f(x)-f(y) \leqq \frac{1}{3^{p+1}} \varepsilon_{0}
$$

ist. Diejenigen Würfel $W_{k_{i} i}\left(i=1, \ldots, 2^{n k_{1}}\right)$, welche in $W_{v_{0}^{\prime} i_{0}}$ enthalten sind, bilden eine Zerlegung ron $W_{r_{n}^{\prime} i_{n}}$. Daher läßt sich Lemma 2.2 anwenden, und wir erhalten auf Grund ron (2.2) einen solchen Teilwürfel $W_{k_{1} i_{1}} \subset W_{r_{u}^{\prime} i_{a}}$, daß nach Ber ücksichtigung von

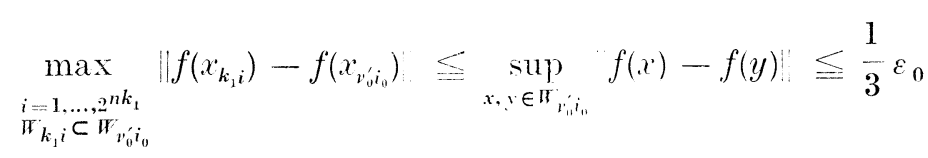

die Ungleichung

$$
r\left(x_{k_{1} i_{1}}, W_{k_{1} i_{1}}\right) \geqq \varepsilon_{0}-\frac{1}{3} \varepsilon_{0}
$$

besteht. Die Anwendung von Lemma 2.2 auf die Zerlegung von $W_{k_{1} i_{1}}$ in die Teilwürfel $W_{k_{2} i}\left(i=1, \ldots, 2^{n k_{s}} \mid W_{k_{i} i} \subset W_{k_{1} i_{1}}\right)$ liefert einen Teilwürfel $W_{k_{2} i_{3}} \subset W_{k_{1} i_{1}}$ mit 


$$
\left\|r\left(x_{k_{2} i_{2}}, W_{k_{2} i_{2}}\right)\right\| \geqq \varepsilon_{0}-\frac{1}{3} \varepsilon_{0}-\frac{1}{3^{2}} \varepsilon_{0}
$$

wenn wir jetzt von (2.4) ausgehen und (2.3) für $v=1$ beachten. Durch Iteration dieser Schlußweise entsteht eine Folge von ineinandergeschachtelten Würfeln $W_{k_{1} i_{1}} \supset W_{k_{1} i_{2}} \supset \ldots \supset W_{k_{v} i_{v}} \supset \ldots$, deren Inhalt

$$
I\left(W_{k_{v} i_{v}}\right)=\frac{1}{2^{n k_{\nu}}} I(W)
$$

gegen Null strebt, wobei nach (2.3) für alle $v=1,2, \ldots$ die Abschätzung

$$
\left\|r\left(x_{k_{\nu} i_{\nu}}, W_{k_{\nu} i_{\nu}}\right)\right\| \geqq \varepsilon_{0}\left(1-\sum_{j=1}^{v} \frac{1}{3^{j}}\right) \geqq \frac{\varepsilon_{0}}{2}>0
$$

gilt. Die Punkte $x_{k_{v} i_{v}}$ konvergieren gegen einen Punkt $\bar{x} \in W$. Damit wird

$$
\begin{gathered}
\left\|r\left(x_{k_{v} i_{v}}, W_{k_{\nu} i_{v}}\right)\right\| \leqq\left\|r\left(x_{k_{v} i_{v}}, W_{k_{v} i_{v}}\right)-r\left(\bar{x}, W_{k_{v} i_{v}}\right)+\right\| r\left(\bar{x}, W_{k_{v} i_{v}}\right) \| \\
\leqq
\end{gathered}
$$

Der vorletzte Term dieser Ungleichung strebt gegen Null wegen der Stetigkeit von $f$ insbesondere in $\bar{x}$. Die Zahlen $\| r\left(\bar{x}, W_{k_{\nu} i_{\nu}} \|_{i}\right.$ bilden eine Nullfolge auf Grund der elementaren Derivierbarkeit von $\omega$ insbesondere in $\bar{x}$, weil die Würfel $W_{k_{\nu} i_{\nu}}$ im Sinne von Definition 1.1 gegen $\bar{x}$ konvergieren und $\bar{x} \in W_{k_{\nu} i_{\nu}}$ für alle $v$ gilt. Daher strebt auch

$$
\left\|r\left(x_{k_{\nu} i_{\nu}}, W_{k_{\nu} i_{\nu}}\right)\right\|
$$

gegen Null im Widerspruch zu (2.5), und der Satz von Stokes ist bewiesen.

Rheinisch-Westfälische T€chnische Hochschule Aachen

Institut für Reine und Angewandte Mathematik

D-5100 Aachen

Deutchland 


\section{Literatur}

[1] Hermans, P.: Über eine Verallgemeinerung der alternierenden Ableitung von Differentialformen. - Universität Jyväskylä, Mathematisches Institut, Bericht 12, 1971.

[1a] - -- Über eine Verallgemeinerung der alternierenden Ableitung von Differentialforinen I. - Math. Nachr. 52, 1972, S. 85-99.

[2] Klemola, T.: Reguläre Mengen von Simplexen und der Satz von Stokes. - Ann. Acad. Sci. Fennicæ A. I. 295, 1961.

[3] MüLler, CL.: Über einen neuen Zugang zur mehrdimensionalen Differential- und Inte. gralrechnung. - Erscheint demnächst.

[4] Nevanlinna, F. und R.: Absolute Analysis. - Grundlehren der mathematischen Wissenschaften 102, Springer-Verlag, Berlin Göttingen Heidelberg, 1959.

[5] Whitney, H.: Geometric integration theory. - [Fourth printing.] Princeton Mathematical Series 21, Princeton University Press. Princeton (New Jersey), 1971. 